\title{
gs \\ Magnetic excitations and femtomagnetism of FeRh: A first-principles study
}

\author{
Leonid M. Sandratskii* \\ Max-Planck-Institut für Mikrostrukturphysik, Weinberg 2, D-06120 Halle, Germany \\ Phivos Mavropoulos \\ Peter Grünberg Institut and Institute for Advanced Simulation, Forschungszentrum Jülich and JARA, D-52425 Jülich, Germany
}

(Received 1 February 2011; published 3 May 2011)

\begin{abstract}
The paper is partly motivated by recent pump-probe experiments with ultrashort laser pulses on antiferromagnetic FeRh that have shown the generation of magnetization within a subpicosecond time scale. On the other hand, the physical mechanism of the thermal antiferromagnetic-ferromagnetic (AFM-FM) phase transition in FeRh, known for many decades, remains a topic of controversial discussions. The selection of the magnetic degrees of freedom as well as the treatment of the magnetic excited states differ strongly in recent models by different authors. We report a density functional theory (DFT) investigation of FeRh. For the study of excited states, DFT calculations with constraints imposed on the directions and values of the atomic moments are employed. We show that the formation of the Rh moment as a consequence of the AFM-FM phase transition cannot be described within the Stoner picture. Instead, an implicit spin splitting of the Rh states takes place in the AFM phase, resulting in the intra-atomic spin polarization of the Rh atoms. This property is a consequence of the strong hybridization between $\mathrm{Rh}$ and $\mathrm{Fe}$ states. The Fe-Rh hybridization is an important factor in the physics of $\mathrm{FeRh}$. We demonstrate that the ferromagnetic Fe-Rh exchange interaction is robust with respect to the crystal volume variation, whereas the antiferromagnetic Fe-Fe exchange interaction is strongly volume dependent. These different volume dependencies of the competing exchange interactions lead to their strong compensation at certain crystal volume. We perform Monte Carlo simulations and show that the calculated thermodynamics depends on the way the magnetic degrees of freedom are selected. We argue that the excited states resulting from the variation of the value of the $\mathrm{Rh}$ moment treated as degree of freedom are important for both the equilibrium thermodynamics of FeRh and the femtomagnetic phenomena in this system. We also study the spin mixing caused by spin-orbit coupling. The obtained value of the Elliott-Yafet spin-mixing parameter is comparable with earlier calculations for the ferromagnetic $3 d$ metals. We draw the conclusion that the Elliott-Yafet mechanism of the angular-momentum transfer between electrons and lattice plays an important role in the femtomagnetic properties of FeRh.
\end{abstract}

DOI: 10.1103/PhysRevB.83.174408

PACS number(s): 75.10.-b, 71.10.Li, 75.70.Tj

\section{INTRODUCTION}

The progress in the development of femtosecond lasers has lead to the emergence of a new branch of solid-state magnetism often referred to as femtosecond magnetism or femtomagnetism. Pump-probe experiments on the elemental $3 d$ ferromagnets have shown that substantial demagnetization takes place within subpicosecond time intervals (see, e.g., Refs. 1-3). Since the angular momentum is one of the conserved physical quantities, the question arises regarding the mechanism of the ultrafast change of the angular momentum of the electron system. Despite much research this question remains the topic of intense debates. Most of the proposed scenaria consider the spin-orbit coupling as a necessary component of the angular-momentum dissipation. ${ }^{4-9}$ Recently, additional arguments have been suggested ${ }^{5}$ supporting one such scenario: the Elliott-Yafet mechanism ${ }^{10,11}$ of angularmomentum transfer between electrons and lattice. The efficiency of the Elliott-Yafet mechanism is determined by the parameter describing the spin mixing of the electron states due to spin-orbit coupling (SOC). The theory, based on first-principles calculations of this parameter, allowed the authors to explain the demagnetization behavior of different ferromagnetic systems. ${ }^{5}$ This point of view was, however, challenged by Battiato et al., ${ }^{12}$ who suggested a very different theoretical model where SOC does not play a role and the quenching of the magnetization appears as the consequence of different scattering properties of the electrons with different spin projections.

In this controversial situation the femtomagnetic phenomena observed in $\mathrm{FeRh}^{13,14}$ becomes increasingly more interesting. In contrast to the ferromagnetic systems with femtosecond demagnetization, one deals here with the opposite effect of ultrafast generation of the magnetization after laser irradiation of the antiferromagnetic FeRh. Since in an antiferromagnet the electron subsystems with opposite spin projections have identical properties, the mechanism of Battiato et al. ${ }^{12}$ does not apply. Therefore, the analysis of the efficiency of the Elliott-Yafet mechanism in femtomagnetism of FeRh promises not only to shed light on the properties of this particular system but also to provide additional arguments in the discussion of the efficiency of various mechanisms of the demagnetization in ferromagnets.

The appearance of a net magnetization after laser irradiation of AFM FeRh is not surprising since the system is known to experience an AFM-FM thermal phase transition at a temperature of $\sim 370 \mathrm{~K} .{ }^{15}$ Therefore, it can be expected that the ultrafast energy supply by the laser light will also lead to the 
transition of the system into the magnetized state. However, since the laser light cannot serve as a source of the angular momentum in the experiments considered ${ }^{16}$ this source must be found within the system.

One purpose of this paper is to investigate the efficiency of the Elliott-Yafet mechanism in the femtomagnetic properties of FeRh. However, this question cannot be addressed without a deep understanding of the physics of the AFM-FM phase transformation. We emphasize the important difference between the femtomagnetic phenomena in ferromagnetic $3 d$ metals and in FeRh: In $3 d$ metals the effect consists of quantitative changes within the same phase, whereas in FeRh a transformation between different phases takes place.

The necessity to deal with phase transformation brings us to another problem where the opinions of different researchers are again very far from a consensus. Although the AFM-FM phase transition in FeRh has been known for many decades ${ }^{17}$ and numerous experimental and theoretical works have been devoted to this system (see, e.g., Refs. 18-22 for theoretical studies based on the DFT), the physical mechanism of this transition remains a matter of debate. In a historical view the first attempts to understand the phase transition were based on Kittel's model that assumes the change of the sign of the effective $\mathrm{Fe}-\mathrm{Fe}$ interaction as a result of the thermal lattice expansion. ${ }^{23}$ This model was nor successful since it does not explain the experimentally detected strong entropy change during the phase transition. ${ }^{24}$

A common point among recent theories based on the results of DFT calculations s $^{13,20,21}$ is the crucial role of the Rh moments in the stabilization of the FM phase. In other respects these three models differ markedly. Gruner et al. ${ }^{20}$ consider both $\mathrm{Fe}$ and $\mathrm{Rh}$ moments as Ising-type one-dimensional spins, whereas Ju et al. ${ }^{13}$ (see also Ref. 22) and Gu and Antropov ${ }^{21}$ treat both $\mathrm{Fe}$ and $\mathrm{Rh}$ moments as Heisenberg-type 3D vectors. On the other hand, the approaches by Ju et al. and Gu and Antropov differ from each other in the selection of the magnetic degrees of freedom, in particular in the treatment of the moments of the $\mathrm{Rh}$ atoms.

Pursuing our purpose to verify the efficiency of the ElliottYafet mechanism in the femtomagnetism of FeRh we will look closer at the electron properties of this system developing a picture that differs in a number of important aspects from the physical pictures suggested earlier. Special attention will be devoted to the comparison with the model of Ju et al. ${ }^{13}$ as this model is suggested in the joint experimental-theoretical paper on femtomagnetism of FeRh and is the only model applied to the interpretation of the femtomagnetic effects.

Since the moments of the $\mathrm{Rh}$ atoms play a crucial role in the phase transformation in $\mathrm{FeRh}$ we will begin with a closer look at the the physical mechanism of the Rh moment formation (Sec. II). In Sec. III we discuss the questions related to the selection of the magnetic degrees of freedom and study the volume dependence of exchange parameters. In Sec. IV, the results of Monte Carlo simulations are reported. In Sec. V, the magnetic states obtained by means of the constraint on the values of the Rh moments are discussed. In Sec. VI the spin-orbit coupling is included in the consideration, and we estimate the parameters important for the Elliott-Yafet mechanism of the angular-momentum exchange between electrons and lattice.
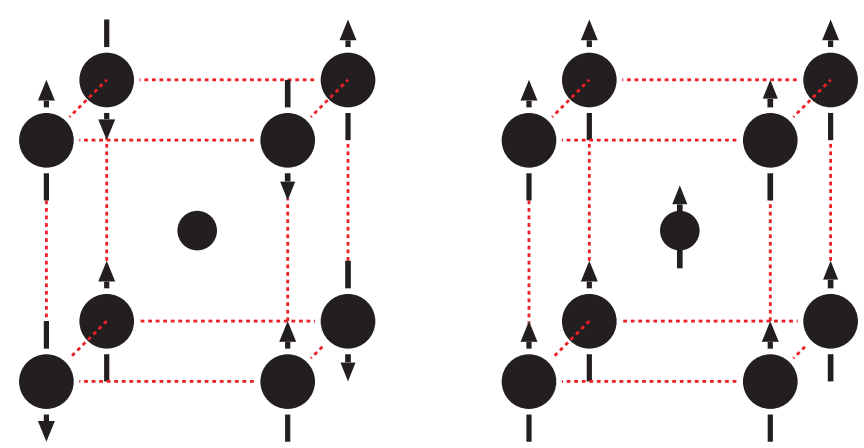

FIG. 1. (Color online) Magnetic structure of AFM (left) and FM (right) FeRh. The corners of the cube host $\mathrm{Fe}$ atoms with a moment of $\sim 3 \mu_{B}$, the center of the cube hosts a Rh atom with zero moment in the AFM phase and $\sim 1 \mu_{B}$ in the FM phase.

\section{NATURE OF Rh MOMENT}

Taking into account the important role played by the $\mathrm{Rh}$ moments in the magnetism of FeRh, ${ }^{13,20-22}$ we begin with the discussion of the physical nature of this moment.

Experimentally it is well established that the Fe moment is close to $3 \mu_{B}$ in both AFM and FM phases, whereas the $\mathrm{Rh}$ moments change from zero in the AFM to about $1 \mu_{B}$ in the FM phase. In the AFM phase, the nearest neighborhood of each $\mathrm{Rh}$ atom contains equal number of $\mathrm{Fe}$ atoms with magnetic moment direction "up" and with direction "down." Therefore no net Rh moment can appear in the AFM state under the influence of the Fe subsystem. The magnetic structure is schematically represented in Fig. 1.

Because of the variation of the $\mathrm{Rh}$ moment from zero in the AFM state to one in the FM state it is seemingly straightforward to interpret the $\mathrm{Rh}$ moment as induced by the Fe moments. Ju et al. ${ }^{13,22}$ suggest invoking the Stoner model to describe the appearance of the Rh moment under the influence of the variation of the $\mathrm{Fe}$ magnetic structure from AFM to FM. The exchange field of the Fe moments at the Rh sites is treated as an external magnetic field with respect to the Rh states. In the AFM phase the Rh states are considered nonmagnetic. We will, however, show that the Stoner model misses important features of the Rh states in FeRh and does not provide an adequate physical picture of the ground and excited states of the system. We will also demonstrate that the notion of the induced moment, although useful for qualitative characterization of some features of the electronic structure of $\mathrm{FeRh}$, cannot be considered as a reason for the reduction of the set of the relevant magnetic excited states, as suggested in Refs. 13 and 22.

The density functional theory calculations reported in this paper were performed within the augmented spherical waves (ASW) method ${ }^{25}$ except in the case of the spin-density plots of the $\mathrm{Rh}$ atom where the calculations were done using the fullpotential Korringa-Kohn-Rostoker Green function method. ${ }^{26}$ The local spin-density approximation ${ }^{27}$ (LSDA) was used for the description of exchange and correlation effects. We will specify the crystal volume used in the calculations by the value of the Wigner-Seitz atomic radius $r_{s}$. The volume of the sphere of this radius corresponds to the volume per atom in the crystal. Throughout the paper, atomic units are used for length ( 1 a.u. $\left.=1 \mathrm{a}_{B}=0.529 \AA\right)$. 


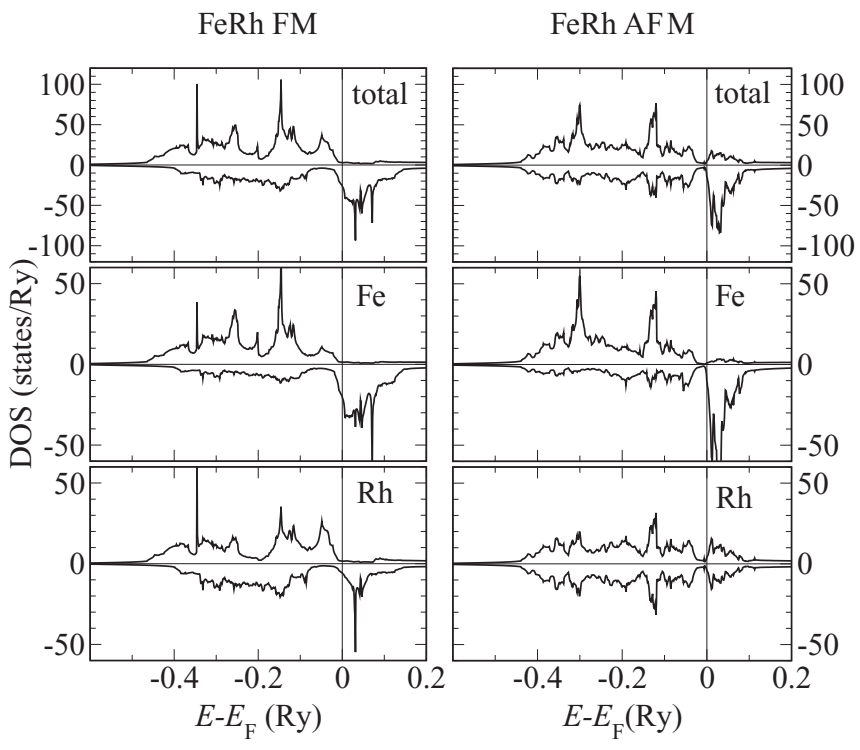

FIG. 2. The DOS of the ferromagnetic (left) and antiferromagnetic (right) state of FeRh. (Upper panel) Total DOS. (Middle panel) Fe-DOS. (Bottom panel) Rh-DOS. In each panel the line with positive DOS values corresponds to the spin-up projection and the line with negative DOS values to the spin-down projection. The total DOS is given per chemical formula unit (FU), the partial atomic DOS per atom.

In Fig. 2(left), the spin-polarized density of states (DOS) of $\mathrm{Fe}$ and $\mathrm{Rh}$ atoms in FM FeRh are presented. The DOS shown is calculated for $r_{S}=2.77$ a.u. close to the theoretical and experimental equilibrium volume. ${ }^{18}$ For each of the atoms the spin-up and spin-down DOS clearly differ, resulting in large atomic magnetic moments: $3.11 \mu_{B}$ for $\mathrm{Fe}$ and $1.07 \mu_{B}$ for $\mathrm{Rh}$. The comparison of the Fe and Rh DOS corresponding to the same spin projection shows that they have a common peak structure. This feature reveals strong hybridization of the $\mathrm{Fe}$ and $\mathrm{Rh}$ states in both spin channels.

In the AFM FeRh (Fig. 2, right panel), the Rh DOS corresponding to the spin-up and spin-down projections are equal. This is an expected result since the $\mathrm{Rh}$ states with a given spin projection hybridize with the locally spin-up states of one of the Fe sublattices and locally spin-down states of the other Fe sublattice. As a consequence, the moment of the Rh atoms is zero, whereas the Fe moment of $2.99 \mu_{B}$ is close to its value in the FM. Remarkably, however, the peak structure of the Rh-DOS in the AFM phase reflects the peak structure of the strongly spin-polarized Fe DOS, just as in the FM case.

This means that Rh electron states do not average the influence of two oppositely magnetized Fe sublattices. Instead, a part of the Rh states with a given spin projection hybridizes mostly with the locally spin-up states of one Fe sublattice, whereas another part of the states with the same spin projection hybridizes mostly with the locally spin-down states of the other Fe sublattice. As a result, the total Rh DOS for the FM and AFM cases are similar to each other and differ substantially from the $\mathrm{Rh}$ DOS of a hypothetical nonmagnetic FeRh (Fig. 3).

A consequence of this implicit spin splitting of the $\mathrm{Rh}$ states is a nonzero intra-atomic spin polarization of the

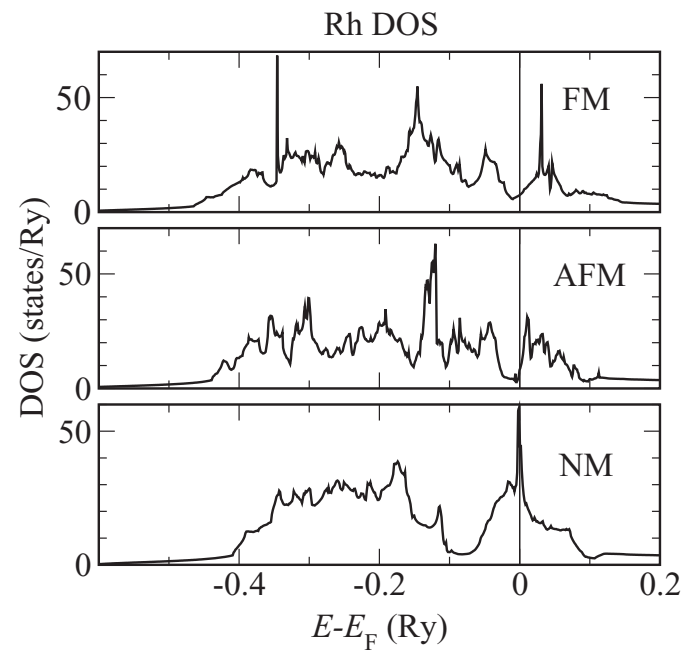

FIG. 3. The partial Rh DOS for ferromagnetic, antiferromagnetic, and nonmagnetic (NM) FeRh. In all cases the DOS presents the sum of the DOS corresponding to both spin components.
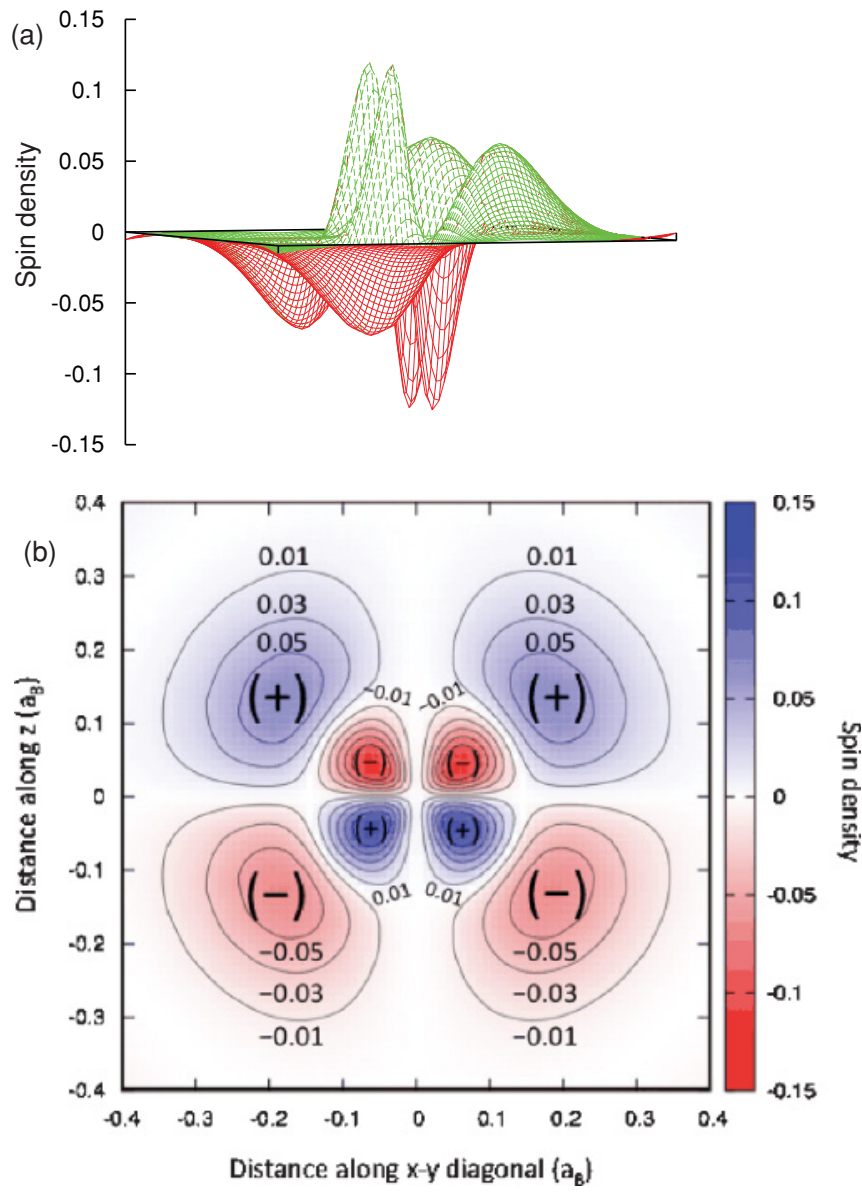

FIG. 4. (Color online) Spin density of the Rh atom in the AFM phase of FeRh. The spin density is shown on a cut along the (110) atomic plane of area $0.4 \times 0.4$ a.u. $^{2}$ around the Rh nucleus. (a) Threedimensional plot demonstrating the peak structure of the spin density. (b) Contour plot of the spin density in the same region demonstrating the symmetry of the positive and negative peaks. 
Rh atom (Fig. 4). The zero value of the total moment of the Rh atoms appears as the result of the compensation of the opposite spin polarizations in different parts of the atomic volume. The implicit spin splitting and intra-atomic spin polarization make the $\mathrm{Rh}$ electron states in the AFM FeRh differ from the nonmagnetic phase of the Stoner theory where the wave functions of the electron states with opposite spin projections are identical and neither of these two features is present.

The implicit spin polarization of the Rh states explains the surprising property of FeRh that a relatively low temperature of $\sim 370 \mathrm{~K}$ is sufficient to transform the state of the $\mathrm{Rh}$ atom with zero moment to the state with the moment of $1 \mu_{B}$. In the Stoner picture the energy difference between the nonmagnetic state and the state with a moment of this magnitude is usually the equivalent of thousands of Kelvins. Indeed, the difference of the energies corresponding to the nonmagnetic and FM states of FeRh is $\sim 56 \mathrm{mRy}(\sim 9000 \mathrm{~K})$ per formula unit compared to the value of $\sim 1 \mathrm{mRy}$ per formula unit for the energy difference between FM and AFM states.

These considerations show that instead of the Stoner-type response of the nonmagnetic $\mathrm{Rh}$ states to the field determined by the net magnetization of the Fe subsystem, the formation of the $\mathrm{Rh}$ moment is governed by the strong covalency with the spin-polarized Fe states. The AFM-FM transition results in a redistribution of the local spin-up and spin-down components in the Rh states transforming the implicit spin splitting in the AFM FeRh to an explicit spin splitting in the FM FeRh and, therefore, leading to the appearance of the net atomic Rh moment.

In the following sections we will show that the study of the volume dependence of the exchange interactions provides interesting information on the properties of the system. Figure 5 presents the calculated volume dependence of the atomic moments. The Fe spin moment increases somewhat with increasing volume in both FM and AFM states. Such

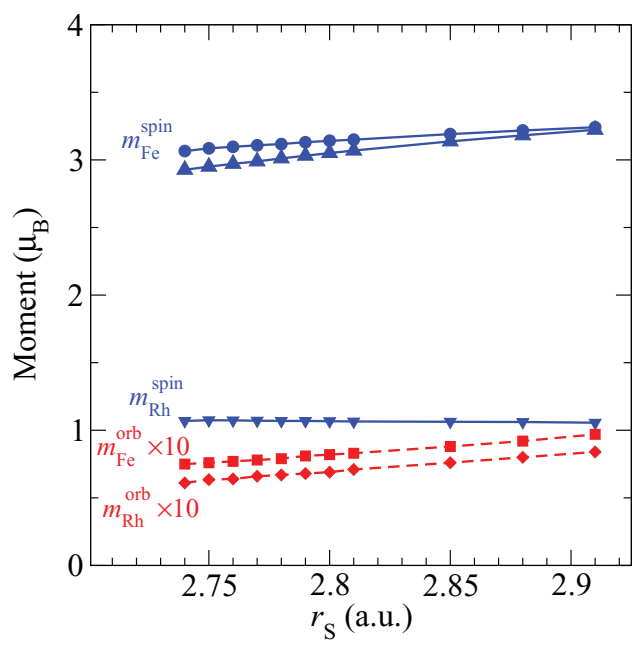

FIG. 5. (Color online) The dependence of atomic moments on crystal volume. Shown are Fe spin moment in the FM (spheres) and AFM (up-triangles) states, Rh spin moment in the FM (downtriangles), Fe orbital moment (squares), and $\mathrm{Rh}$ orbital moment in the FM (diamonds). The Fe orbital moment in the AFM practically coincides with the corresponding curve in the FM and is not shown. For $\mathrm{Rh}$, both spin and orbital moments in the AFM are zero. behavior is expected as a consequence of the band narrowing accompanying the increase of the volume. The $\mathrm{Rh}$ spin moment in the FM phase remains practically unchanged, reflecting the combined influence of the increasing exchange splitting of the Fe states and decreasing hybridization of the Fe and Rh states.

Further insights into the nature of the Rh moment will be gained in the next section where we discuss excited magnetic states.

\section{CONSTRAINED CALCULATIONS AND EXCITED STATES}

\section{A. General principles}

From the microscopic point of view the thermal behavior of a system is determined by the spectrum of excited states. Therefore an adequate treatment of the excited states is crucial for the understanding of the processes at nonzero temperatures. An important step in the consideration of the excited states of a given system is the selection of the magnetic degrees of freedom determining the excited states. In this respect it is remarkable that the models of FeRh based on the DFT calculations differ in how the excited states are treated. Not only the selection of the degrees of freedom but also the treatment of the excited states corresponding to the same degrees of freedom differ strongly. ${ }^{13,20-22}$

In the model by $\mathrm{Gu}$ and Antropov ${ }^{21}$ the excitations correspond to the excited states of the Heisenberg Hamiltonian. In the AFM phase this is the Hamiltonian of the Fe moments only, whereas in the FM phase it is the Hamiltonian of the $\mathrm{Fe}$ and Rh moments. Neither the appearance of nonzero Rh moments in excited states of the AFM phase nor the variation of the value of the Rh moments in excitations of the FM phase is taken into account. In the model by $\mathrm{Ju}$ et al., ${ }^{13,22}$ only the directions of the Fe moments are considered as degrees of freedom. On the other hand, the formation of $\mathrm{Rh}$ moments in the AFM phase and the variation of the Rh-moment value in the FM phase are taken into account by demanding that the moment of each $\mathrm{Rh}$ atom is determined by the directions of the neighboring Fe moments: $\vec{m}_{\mathrm{Rh}}=\chi \sum_{i} \vec{m}_{\mathrm{Fe}}^{i}$, with $\chi$ a constant and $i$ running over the nearest neighbors. Thus, the appearance of the Rh moment in the AFM phase caused by the disordering of the Fe moments is considered by $\mathrm{Ju}$ et al. and is argued to be the physical mechanism of the AFM-FM phase transition.

The work by Gruner et al., ${ }^{20}$ although most advanced in the statistical mechanics study of the model, treats all atomic moments as one-dimensional Ising spins, which complicates the comparison of the results of this study with experiment and our calculations. Therefore, in the analysis of our data we will mostly refer to the models by Ju et al. and Gu and Antropov.

In view of the strong difference between the models based on the DFT calculations it is worthwhile to briefly formulate the general principles of the treatment of the excited magnetic states within the DFT.

The consideration of the energy response to the variation of the charge and spin densities is at the heart of the density functional theory. The minimization of the energy with respect to the variations of the spin density gives the magnetic 
ground state of the system. To perform the calculation of an excited state, the energy functional must be minimized under certain constraining conditions specifying this state. The mathematical procedure of constrained minimization results in the introduction of an external magnetic field stabilizing the desired magnetic state. ${ }^{28}$ The directions of the moments are stabilized by the fields orthogonal to these directions, whereas the values of the moments are stabilized by the fields parallel to the moments. Therefore, in a general treatment, all states obtained under constraint on the directions and values of the atomic moments should be considered as possible excited states of the system. Turning back to FeRh, we conclude that within this general scheme there is no basis for a priori reduction of the magnetic degrees of freedom to the directions of the Fe moments as well as for the neglect of the variation of the value of the Rh moment in the excited states of the FM and AFM phases.

The calculation of a complete set of constrained states of a solid is a problem of formidable complexity and cannot be solved in a direct way. The possible approximations involve the restrictions of both the number of excited states studied by the DFT methods and the accuracy of the study of the selected excited states. It is worth noting here that the calculation of the excited states is not a final purpose of a physical study. This information is needed in the statistical mechanics investigation of the system outside the ground state. Therefore one needs to deal with a compromise between, on the one hand, the necessity of a sufficiently simple parametrization of the excited states in a model magnetic Hamiltonian to make the statistical mechanics calculations feasible and, on the other hand, the necessity to take into consideration all physically relevant features of the excited states.

Coming back to the models of Refs. 13 and 20-22 we can characterize the selection of constrained states adopted in these models as follows. Ju et al. ${ }^{13,22}$ suggest restricting the consideration of the excited states to the states determined by the constrained directions of the Fe moments. The states obtained by constraining the value of the Fe moments and by any constraint on the $\mathrm{Rh}$ moments are excluded from the consideration. The argumentation for this restricted choice of the excited states is based on the induced nature of the $\mathrm{Rh}$ moments. However, in the previous section we have shown that the feature that can be referred to as induced nature of the $\mathrm{Rh}$ moment reflects strong hybridization between $\mathrm{Rh}$ and $\mathrm{Fe}$ states and by itself imposes no restrictions on the set of excited states or on their relative importance. In Sec. IV we study the influence of the selection of degrees of freedom on magnetic thermodynamics. In Sec. V we argue that the account for only the states determined by the constraint on the directions of the Fe moments excludes from the consideration the excitations important for the interpretation of femtomagnetic experiments.

$\mathrm{Gu}$ and Antropov ${ }^{21}$ calculate the exchange parameters by the estimation of the energy price of the infinitesimal deviations of the directions of atomic moments from an equilibrium magnetic configuration. ${ }^{29}$ In these calculations the constraint is imposed on the directions of the atomic moments. The DFT calculation of the energy differences is based on the so-called magnetic force theorem and reduces to the evaluation of the band part of the energy. The appearance of the $\mathrm{Rh}$ moment in the AFM phase or the variation of the value of the Rh moment in the FM phase are not considered. Finally, Gruner et al. ${ }^{20}$ perform DFT calculations only for collinear FM and AFM states. This means that constraints are imposed on the directions of the atomic moments. Because of the symmetry properties of both collinear structures they do not need effective magnetic fields for their stabilization. We deal in this case with a symmetry constraint. ${ }^{30}$

\section{B. Constrained calculations}

We begin the consideration of the excited states with a simple constrained calculation that provides information on the exchange interactions between $\mathrm{Fe}$ and $\mathrm{Rh}$ moments. We start with the ferromagnetic FeRh and, keeping both $\mathrm{Fe}$ and $\mathrm{Rh}$ subsystems to be separately ferromagnetic, vary the angle between $\mathrm{Fe}$ and $\mathrm{Rh}$ moments from $0^{\circ}$ to $90^{\circ}$. For each angle a self-consistent calculation is performed. The result of such calculations is presented in Fig. 6. It is similar to the corresponding result discussed by $\mathrm{Ju}$ et $a l .{ }^{13}$ and presented by Mryasov. ${ }^{22}$ For the sake of comparison of different approaches it is worth noting that, although $\mathrm{Ju}$ et al. use such configurations to study exchange interactions between $\mathrm{Fe}$ and $\mathrm{Rh}$ moments, they are not taken into account in the discussion of statistical mechanics. Indeed, if the constraint on the angle between the directions of the $\mathrm{Rh}$ and Fe moments is removed the configurations shown in Fig. 6 become unstable and the DFT calculation started with arbitrary initial $\theta$ results in the same collinear ferromagnetic state. According to the general approach to the excited states within DFT there is no basis for this variance in the treatment of the constrained states, namely to consider them in the calculation of exchange parameters but exclude them from the magnetic excitations contributing to the statistical mechanics of the system.

We see (Fig. 6) that the value of the Fe moment depends very weakly on $\theta$, whereas the Rh moment decreases monotonously from the value close to $1 \mu_{B}$ to zero. In a rough approximation $m_{\mathrm{Rh}}(\theta)$ is proportional to $\cos \theta$. The strong increase of the energy of the system with increasing $\theta$ reveals a ferromagnetic exchange interaction between $\mathrm{Fe}$ and $\mathrm{Rh}$ moments. Since the energy curve is roughly proportional to $\left(m_{\mathrm{Rh}}^{0}\right)^{2}-\left[m_{\mathrm{Rh}}(\theta)\right]^{2}, \mathrm{Ju}$ et al. ${ }^{13,22}$ suggest treating this energy contribution as the Stoner energy of the Rh subsystem. Our energy curve is very close to the curve reported by Mryasov. ${ }^{22}$ However, in this case we also consider the reference to the Stoner model as physically not well founded. The same type of the $\theta$ dependence follows from a Heisenberg-type bilinear interaction between $\mathrm{Fe}$ and $\mathrm{Rh}$ moments $J_{\mathrm{FeRh}} \mathbf{m}_{\mathrm{Fe}} \cdot \mathbf{m}_{\mathrm{Rh}}$ if the dependence of $m_{\mathrm{Rh}}$ on angle $\theta$ between $\mathrm{Fe}$ and $\mathrm{Rh}$ moments is taken into account. Since the estimation of the Stoner parameter ${ }^{31}$ of the $\mathrm{Rh}$ atom gives a value of $50 \mathrm{mRy}$ that is much larger than the parameter evaluated from the calculations presented in Fig. 6 we conclude again that the conventional Stoner picture is not adequate to describe the magnetism of the Rh atoms in FeRh.

The dashed energy curve presented in the bottom panel of Fig. 6 is obtained by applying the magnetic-force theorem. ${ }^{29}$ This theorem suggests that the energy price of the deviation of the atomic moments from the ground-state directions can be estimated as the difference between band energies of the ground and excited magnetic configurations calculated with the use of the same ground-state atomic potentials. Therefore, 
in this calculation the dependence of the $\mathrm{Rh}$ moment on angle $\theta$ between $\mathrm{Fe}$ and $\mathrm{Rh}$ moments is not taken into account. The curve obtained with the magnetic-force-theorem calculation gives the estimation of the parameter of the exchange interaction between $\mathrm{Fe}$ and $\mathrm{Rh}$ moments $J_{\mathrm{Fe}-\mathrm{Rh}}$ $\sim 1.2 \mathrm{mRy}$.

To describe the energy dependence obtained selfconsistently (bottom panel of Fig. 6, full curve), we need two terms. The first term gives the exchange interaction between Fe and Rh moments

$$
H_{\mathrm{FeRh}}=-\sum_{i, v} J_{\mathrm{FeRh}}^{i v} \mathbf{e}_{i} \mathbf{m}_{\nu},
$$

where $i$ runs over the $\mathrm{Fe}$ atoms and $v$ runs over the Rh atoms. Here $\mathbf{e}_{i}$ is the unit vector in the direction of the magnetic moment of the $i$ th atom and $\mathbf{m}_{v}$ gives the magnetic moment of the $v$ th $\mathrm{Rh}$ atom. In the ferromagnetic state $m_{\mathrm{Rh}} \approx 1 \mu_{\mathrm{B}}$. In the form of the Hamiltonian given by Eq. (1) the values of the $\mathrm{Fe}$ atomic moments are included in the definition of the exchange parameters $J_{\mathrm{FeRh}}^{i v}$. Since the values of the Fe moments do not vary during simulations, this form of the Hamiltonian is convenient. It is, however, essential for the simulations that the $\mathrm{Rh}$ moments can assume different values. The Fe-Rh exchange parameter remains unchanged and valid for all values of the Rh moment in Eq. (1). Only the interaction between nearest $\mathrm{Fe}$ and $\mathrm{Rh}$ atoms is considered, since all estimations show that the interactions between more distant neighbors are much weaker.

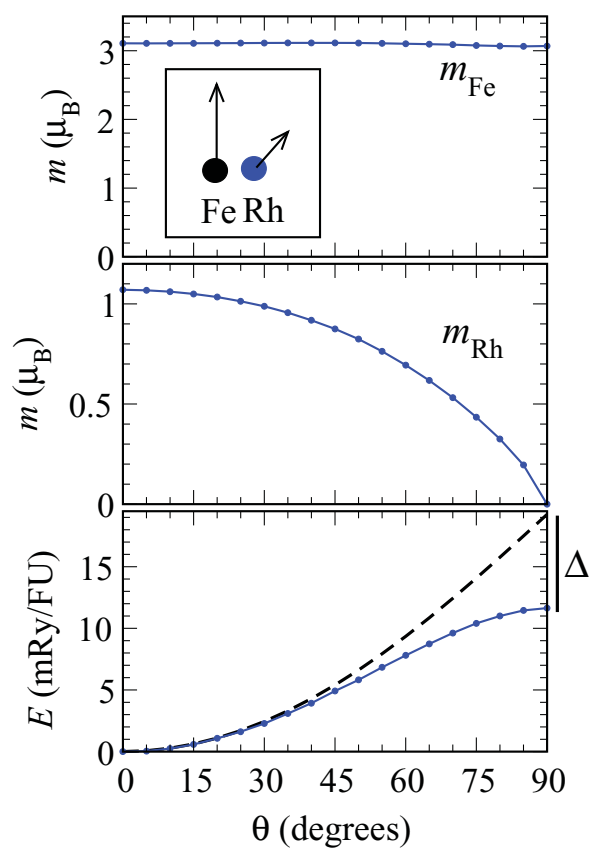

FIG. 6. (Color online) Angle dependence of the Fe moment, Rh moment, and energy of the state. The angle between $\mathrm{Fe}$ and $\mathrm{Rh}$ moments is $\theta$. Both Fe and Rh sublattices are kept ferromagnetic (see insert in the upper panel). The broken energy curve in the bottom panel is the result of the non-self-consistent calculation based on the magnetic force theorem that does not take into account the change of the Rh moment (see the text). $\Delta$ in the bottom panel marks the on-site energy contribution [Eq. (2)].
The second energy term,

$$
H_{\mathrm{Rh}}=\sum_{v} J_{\text {on-site }} m_{v}^{2},
$$

does not have an explicit dependence on the Fe moment and can be treated as the change of the energy of the Rh atom caused by the variation of the Rh atomic moment. The estimation on the basis of two energy curves in the bottom part of Fig. 6 gives $J_{\text {on-site }} \sim 6 \mathrm{mRy}$.

Note that two contributions [Eqs. (1) and (2)] have opposite types of the variation with the change of the Rh moment. The bilinear term assumes the lowest value for the maximal projection of the $\mathrm{Rh}$ moment on the exchange field of the $\mathrm{Fe}$ environment, whereas the on-site term is minimal for the zero value of the Rh moment. These contributions will be discussed in more detail in Sec. IV.

An interesting piece of information about the system is obtained by the study of the volume dependence of the exchange interactions. When the calculations of the type presented in Fig. 6 are performed for larger atomic volumes, they show a weak volume dependence that reveals the robustness of the $\mathrm{Fe}-\mathrm{Rh}$ exchange interaction with respect to the change of the volume.

The configurations considered above (Fig. 6) do not provide information on the $\mathrm{Fe}-\mathrm{Fe}$ exchange interactions since the $\mathrm{Fe}$ moments remain parallel to each other. Next we consider a type of magnetic state that, although still rather simple, contains information on the interaction of the Fe moments belonging to different sublattices. We begin with the AFM structure and assume the $\mathrm{Fe}$ moments to be collinear to the $x$ axis. The constrained states are obtained by rotating the moments of the two Fe sublattices toward the $z$ axis by the same angle up to the ferromagnetic state (Fig. 7). The angle $\theta$ between the $\mathrm{Fe}$ moments and the $z$ axis varies from $90^{\circ}$ to $0^{\circ}$. At $\theta=0$ the system becomes ferromagnetic. There is a number of interesting observations that follow from these calculations, which are performed at two different crystal volumes.

The lowest energy corresponds to the AFM structure in agreement with experiment. The shape of the $\theta$ dependence varies strongly with the change of the volume. In Fig. 7 we present the results of calculations for two different volumes. For the smaller volume (Fig. 7, left), the energy curve is monotonous with a minimum at the AFM state and maximum at the FM state. With increasing volume the maximum at $\theta=0$ (FM state) is replaced by local minimum (Fig. 7, right). The energy difference between AFM and FM states decreases with increasing volume. We obtain a kind of metamagnetic situation with a global minimum at the AFM state and a local minimum at the FM state. On the basis of the experimental information about thermal AFM-FM phase transition such a two-minima energy landscape should be considered to be in better correspondence to the experimental data than the smaller-volume monotonous curve. Apparently, the LSDA overestimates the energy difference between the AFM and FM states at the equilibrium volume. $\mathrm{Gu}$ and Antropov also came to this conclusion and have shown that the nonlocal correction to the energy functional suggested by Langreth and $\mathrm{Mehl}^{32}$ improves the situation and makes the FM state energetically stable with respect to small deviations of the atomic moments from the parallel orientation for the crystal volume close to 
the experimental one. It is an interesting problem to compare the performance of different types of DFT schemes beyond LSDA in the case of FeRh. In this paper, however, we restrict the investigation to the LSDA, augmenting it with the study of the volume dependence of the exchange interactions. As already mentioned, this procedure allows us to study different magnetic-energy landscapes, from those at smaller volumes that are far from the experimental situation to the landscapes at larger volumes that are in good correlation with experiment.

For the magnetic configurations presented in Fig. 7, both $\mathrm{Fe}-\mathrm{Rh}$ and $\mathrm{Fe}-\mathrm{Fe}$ interactions contribute to the $\theta$ dependence of the energy since with the variation of $\theta$ the angles between $\mathrm{Fe}$ and $\mathrm{Rh}$ moments as well as between $\mathrm{Fe}$ moments of different sublattices vary. Taking into account the weak volume dependence of the Fe-Rh interactions established above we can conclude that the volume dependence of the $E(\theta)$ curve is a consequence of the strong volume dependence of the exchange interactions between atoms of different Fe sublattices.

To verify this point we consider the energy of the magnetic states where the Fe moments form a configuration identical to the one shown in Fig. 7 while the Rh moments are zero (see inset in Fig. 8). Technically, these calculations are performed by constraining the direction of the $\mathrm{Rh}$ moments to be orthogonal to the plane of the Fe moments, immediately leading to their zero value. The corresponding energy curves are marked as "Fe-Fe" in Fig. 8, denoting that they are determined by the Fe-Fe exchange interactions only. Although the cosine-type shape of the both Fe-Fe curves is similar at the two volumes, the scales differ strongly. The maximum values at $\theta=0$ are 17.3 and $11.7 \mathrm{mRy}$, respectively, for the atomic radii of 2.77 and 2.91 a.u.. The difference of these two energies, $5.6 \mathrm{mRy}$, is very close to the difference of energies calculated
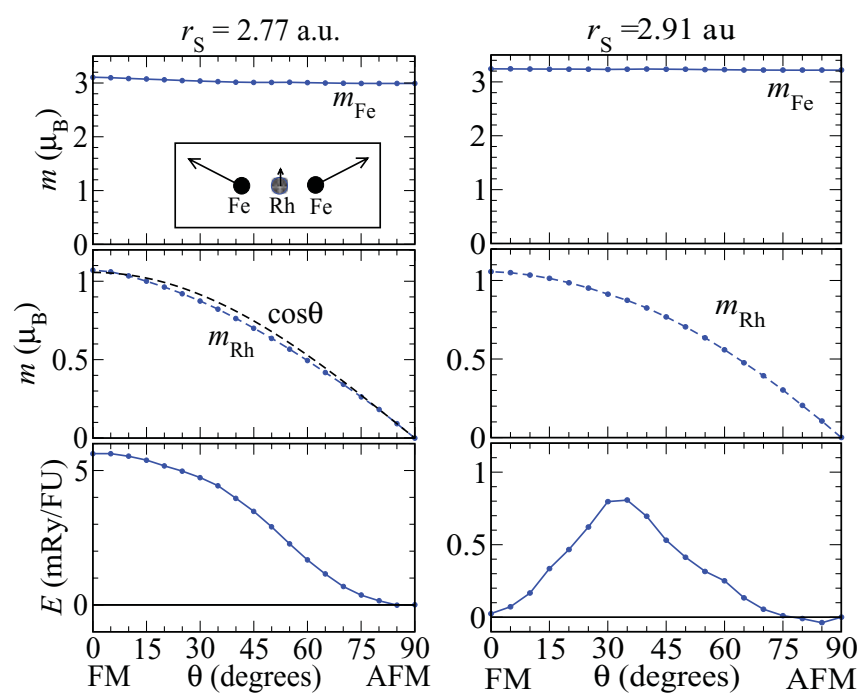

FIG. 7. (Color online) Angle dependence of the Fe moment, Rh moment, and energy of the state. The angle between $\mathrm{Fe}$ and $\mathrm{Rh}$ moments is $\theta$. The magnetic configurations used in the calculations are schematically presented in the insert in the upper panel. The calculations are performed at a lattice constant corresponding to $r_{S}=$ 2.77 a.u. (left) and $r_{S}=2.91$ a.u. (right). Note the difference in energy scale between the two cases.
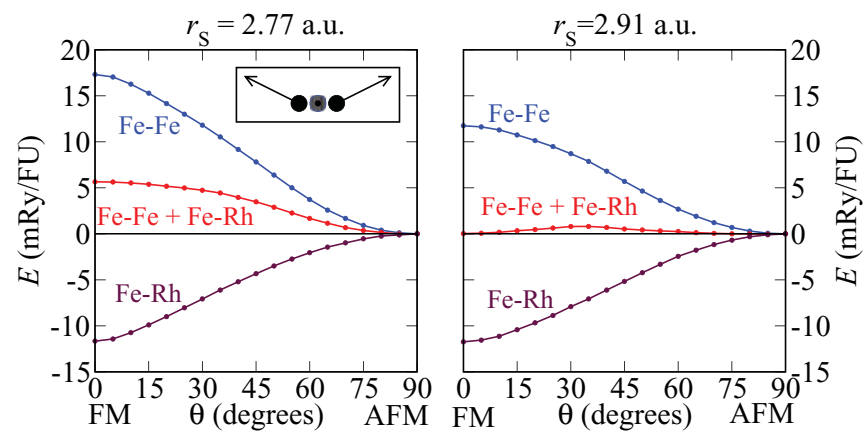

FIG. 8. (Color online) Separation of the contributions of the Fe$\mathrm{Fe}$ and $\mathrm{Fe}-\mathrm{Rh}$ exchange interactions in the energy of the magnetic configurations presented in Fig. 7 for $r_{S}=2.77$ a.u. (left) and for $r_{S}=2.91$ a.u. (right). In the inset, the magnetic configuration with zero $\mathrm{Rh}$ moment is schematically depicted.

with nonzero Rh moments (Fig. 7). The corresponding energy curves are reproduced in Fig. 8 as "Fe-Fe $+\mathrm{Fe}-\mathrm{Rh}$ " curves. This notation emphasizes that both the $\mathrm{Fe}-\mathrm{Fe}$ and $\mathrm{Fe}-\mathrm{Rh}$ exchange interactions contribute to the form of the curves.

Subtracting "Fe-Fe" curves from the "Fe-Fe + Fe-Rh" curves, we obtain the contribution to the energy from the Fe-Rh exchange interaction. Comparison of Fig. 8 and Fig. 6 shows that the Fe-Rh exchange interaction is very robust with respect to both the crystal volume and the magnetic configurations used in the calculations.

Thus, our calculations lead to the conclusion that the ferromagnetic Fe-Rh exchange interaction is weakly volume dependent, whereas the antiferromagnetic intersublattice Fe-Fe exchange interaction is strongly volume dependent and becomes weaker for larger volumes.

For $r_{S}=2.91$ a.u. the Fe-Rh and Fe-Fe exchange interactions strongly compensate each other, resulting in a nonmonotonous " $\mathrm{Fe}-\mathrm{Fe}+\mathrm{Fe}-\mathrm{Rh}$ " energy curve. The different volume dependencies of two competing interactions leads to their strong compensation at a certain volume. This finding reveals the physical origin of the specific properties of FeRh as a competition between AFM and FM phases. The LSDA allows us to establish this important property, although the volume where the strong compensation takes place is larger than the experimental or theoretical equilibrium volume.

The calculations presented in Fig. 8 provide the information about the exchange interactions between $\mathrm{Fe}$ sublattices in a cumulative form. To resolve individual interatomic exchange interactions, we employed the frozen-magnon technique described in earlier publications (see, e.g., Ref. 33) and based on the calculation of spin-spiral configurations for various wave vectors accompanied by a back-Fourier transformation of the energy functions. The Fe-Fe interatomic exchange parameters are shown in Fig. 9. The leading inter-sublattice exchange interactions are antiferromagnetic interactions $J_{\mathrm{FeFe}}[100]$ and $J_{\mathrm{FeFe}}[111]$, where [100] and [111] are the vectors separating $\mathrm{Fe}$ atoms and expressed in the units of the cubic lattice constant. These exchange parameters depend strongly on the atomic volume. For larger volume the interactions become less antiferromagnetic, preserving the negative sign and decreasing in absolute value. 


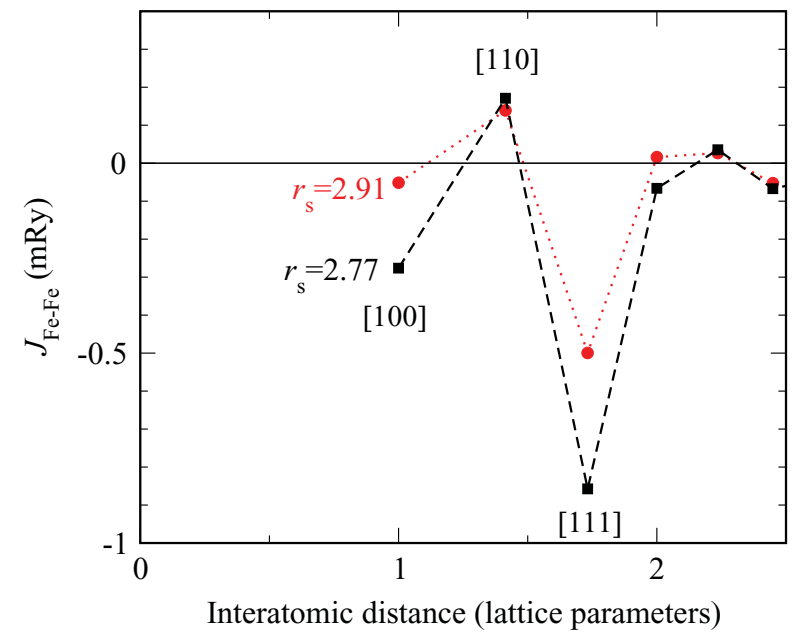

FIG. 9. (Color online) Fe-Fe exchange parameters for two crystal volumes. The exchange parameters are obtained with frozen-moment technique with the ferromagnetic configuration as a starting magnetic state.

\section{MONTE CARLO SIMULATIONS}

As mentioned above, the previous works differ strongly in both the selection of the degrees of freedom and in the treatment of the excited states. Ju et al. ${ }^{13,22}$ consider the directions of the Fe moments as the only degrees of freedom in both FM and AFM phases. The values and directions of the $\mathrm{Rh}$ atomic moments are uniquely defined by the magnetic configuration of the Fe environment: A Rh moment is proportional to the exchange field generated by the the nearest Fe moments at the position of the $\mathrm{Rh}$ atom. According to $\mathrm{Ju}$ et al. it is crucial for the understanding the AFM-FM transition to take into account the appearance of the atomic $\mathrm{Rh}$ moments in the AFM phase. They argue that the Rh moments in the AFM phase drive the phase transition by means of inducing an additional effective $\mathrm{Fe}-\mathrm{Fe}$ interaction.

On the other hand, Gu and Antropov neglect the appearance of the $\mathrm{Rh}$ moments in the AFM phase and consider these moments as Heisenberg degrees of freedom in the FM phase. They came to the conclusion that the driving force of the phase transition differs temperature dependence of the entropy of the AFM and FM phases.

On the basis of general principles of the treatment of the excited magnetic states within DFT (see Sec. III A), we conclude that the consequent approach to the statistical mechanics of FeRh should include features of both models: the $\mathrm{Rh}$ moments should be considered as degrees of freedom and the variation of the value of the Rh moments in both AFM and FM phases ought to be taken into account. Also the excited states determined by the constraints on the values of the atomic moments cannot be a priori excluded. The latter states determine the longitudinal magnetic fluctuations for given directions of the atomic moments (see Sec. V). Since in the general formulation the problem becomes very complex the application of simplified models is an important and inevitable step. This step, however, should be accompanied by the investigation of the physical consequences of the adopted approximations.
The purpose of the Monte Carlo simulations presented in this section is the investigation of the influence of different treatments of the $\mathrm{Rh}$ moments on the thermodynamics of FeRh. We performed a number of simulations based on different types of models. In some of the simulations only the Fe-Fe exchange intaractions were taken into account. The corresponding Hamiltonian has the form

$$
H_{\mathrm{FeFe}}=-\sum_{i, j} J_{\mathrm{FeFe}}^{i j} \mathbf{e}_{i} \cdot \mathbf{e}_{j},
$$

where the sum runs over pairs of $\mathrm{Fe}$ moments and $\mathbf{e}_{i}$ is the unit vector in the direction of the magnetic moment of the $i$ th atom. Similarly to Eq. (1), in the form of the Hamiltonian given by $\mathrm{Eq}$. (3) the values of the $\mathrm{Fe}$ atomic moments are included in the definition of the $\mathrm{Fe}-\mathrm{Fe}$ exchange parameters. Three leading $\mathrm{Fe}-\mathrm{Fe}$ exchange parameters were considered. They correspond to the pairs of the Fe atoms separated by the vectors [100], [110], and [111] (Fig. 9).

In other simulations also the $\mathrm{Rh}$ moment is considered either as a usual Heisenberg variable with a constant value or as an induced moment with the value depending on the $\mathrm{Fe}$ environment. The corresponding energy contributions are evaluated using Eqs. (1) and (2). In the simulations we will use the Fe-Fe exchange parameters for two different values of $r_{S}$ (Fig. 9). Since the Fe-Rh exchange parameter and the $\mathrm{Rh}$ on-site exchange parameter can be treated as volume independent, this will allow us to study the trends due to the variation of the relative strength of various interactions.

We begin with the discussion of the calculations performed with the $\mathrm{Fe}-\mathrm{Fe}$ exchange parameters corresponding to $r_{S}=2.77$ a.u.. If the formation of the $\mathrm{Rh}$ moment is neglected and only Fe-Fe exchange interactions are taken into account we obtain the AFM ground state of the system and very high Neel temperature of $\sim 1000 \mathrm{~K}$ [Fig. 10(a)].

In the next step we took into account the appearance of the nonzero Rh moment in the excited states. In this case a Monte Carlo step consists of several substeps: (i) a new direction of an Fe moment is determined according to a selected pair of random numbers, (ii) the values of the induced $\mathrm{Rh}$ moments are calculated on the basis of the new local Fe environment, and (iii) the energy of the magnetic configuration obtained in this way is calculated accounting for Fe-Fe [Eq. (3)], Fe- Rh [Eq. (1)], and on-site Rh exchange interactions [Eq. (2)]. The acceptance of the configuration takes place according to the Metropolis algorithm. ${ }^{34}$

If only the bilinear $\mathrm{Fe}-\mathrm{Rh}$ interaction, but not the $\mathrm{Rh}$ on-site term, is taken into account, the simulation leads to the ferromagnetic ground state. This, on the one hand, demonstrates the strength of the Fe-Rh interaction and, on the other hand, shows the necessity of careful dealing with the treatment of the effects of the induced moment. Actually, the transformation of the AFM with high Neel temperature to FM is not surprising in this case. The energy difference between the FM and AFM states is about $6 \mathrm{mRy}$ per formula unit (Fig. 7) for $r_{S}=2.77$ a.u.. The neglect of the on-site term with exchange parameter of $6 \mathrm{mRy}$ decreases the energy of the FM state with respect to the AFM state by this value.

If the on-site term is taken into account, the ground state becomes AFM again [Fig. 10(b)]. The Neel temperature is $\sim 800 \mathrm{~K}$, which is $200 \mathrm{~K}$ lower than the Neel temperature 

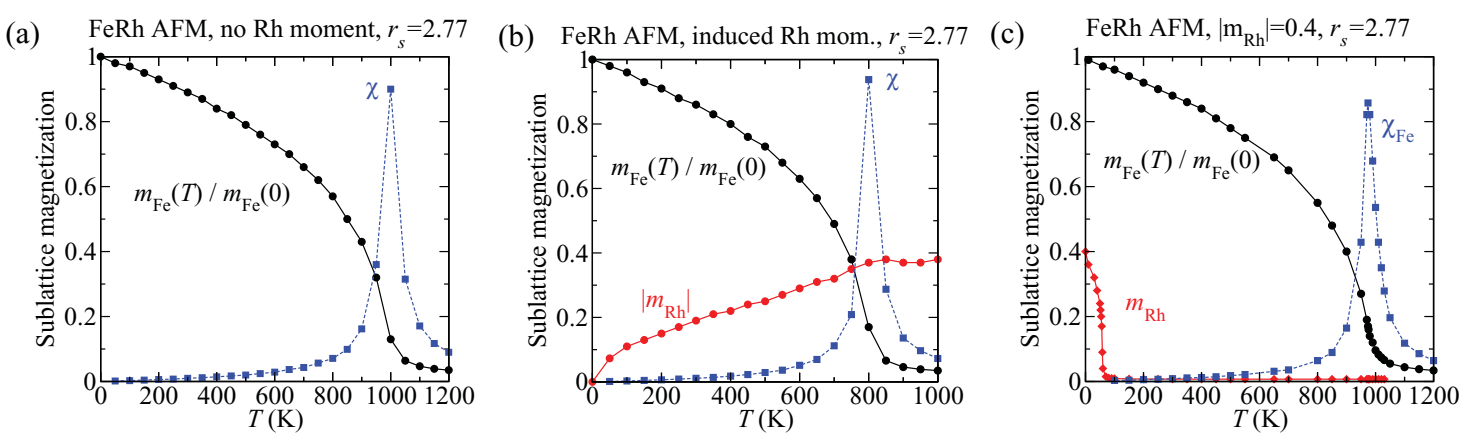

FIG. 10. (Color online) Results of Monte Carlo simulations for the AFM phase of FeRh under different assumptions. The Fe-Fe exchange parameters for $r_{S}=2.77$ a.u. are used in the calculations. The magnetization curves corresponding to Fe give the sublattice magnetization in units of the $\mathrm{Fe}$ atomic moment, whereas the data related to the $\mathrm{Rh}$ magnetic moment are in units of $\mu_{B}$. Magnetic susceptibilities are given in arbitrary units. (a) The generation of the Rh moment is not taken into account. (b) An induced Rh moment taken into account. Only the directions of the Fe moments are treated as degrees of freedom, whereas the Rh moment is tightly related to the Fe magnetic configuration. The curve labeled with $\left|m_{\mathrm{Rh}}\right|$ gives the average of the absolute values of the Rh moments in units of $\mu_{B}$. (c) Both Fe and Rh moments are treated as Heisenberg degrees of freedom. The of the Rh atomic moment has a constant value of $0.4 \mu_{B}$.

obtained with the neglect of the induced $\mathrm{Rh}$ moments. The decrease of the Neel temperature is an expected property since both types of simulations have the same degrees of freedom, that is, the directions of the Fe moments, and the appearance of the Rh moments decreases the energy of the corresponding excited states.

The absolute value of the $\mathrm{Rh}$ moment increases monotonously with increasing temperature and saturates in the paramagnetic region at the value of about $0.4 \mu_{B}$ [Fig. 10(b)]. Next, we performed the simulation considering both $\mathrm{Fe}$ and $\mathrm{Rh}$ moments as Heisenberg degrees of freedom and taking the value of the $\mathrm{Rh}$ moment to be equal to $0.4 \mu_{B}$ in order to investigate the relative importance of two factors on the magnetic thermodynamics: (i) the energies of the excitations and (ii) the number of the excitations governed by the selection of the degress of freedom. In this case [Fig. 10(c)] the disordering of the Rh sublattice was practically completed at $80 \mathrm{~K}$, whereas the Neel temperature of the system increased again to the value of $1000 \mathrm{~K} .{ }^{35}$ This demonstrates that the choice of the degrees of freedom is a very important factor of the model.

Finally, attempting to describe the ferromagnetic state, we performed the latter-type simulation with the experimental value of the $\mathrm{Rh}$ moment of $1 \mu_{B}$. The ground state in this case consists of the collinear ferromagnetic sublattice of the $\mathrm{Rh}$ moments and noncollinear ferromagnetic subsystem of the Fe moments. The disordering takes place at low temperature of $370 \mathrm{~K}$ (not shown).

Now we turn to the simulations with $\mathrm{Fe}-\mathrm{Fe}$ exchange parameters obtained for $r_{S}=2.91$ a.u. (see Fig. 11). If the appearance of the Rh moment is neglected the system is AFM with the Neel temperature of $550 \mathrm{~K}$. Treating the Rh moment of $1 \mu_{B}$ as Heisenberg variable we get a FM ground state and a Curie temperature of $600 \mathrm{~K}$ that is in good correlation with the experimental value of $\sim 670 \mathrm{~K} .{ }^{36}$ If the $\mathrm{Rh}$ moment is treated as induced and only the bilinear Fe-Rh interaction [Eq. (1)] is taken into account, the Curie temperature increases to a very large value of $1260 \mathrm{~K}$. If both bilinear and on-site [Eq. (2)] terms are considered the Curie temperature becomes $760 \mathrm{~K}$.
A number of conclusions can be drawn on the basis of the Monte Carlo simulations. (a) The less antiferromagnetic Fe-Fe exchange parameters obtained for $r_{S}=2.91$ a.u. give better correlations with experiment than the parameters obtained for $r_{S}=2.77$ a.u. This result is expected since the two-minima energy curve (Fig. 7) corresponding to $r_{S}=2.91$ a.u. is in better correlation with experiment than the monotonous energy curve for $r_{S}=2.77$ a.u. (b) The simulations in the model used by Guo and Antropov that neglects the appearance of the $\mathrm{Rh}$ moment in the AFM phase and the change of the values of the $\mathrm{Rh}$ moments in the FM phase give reasonable values of the critical temperatures. However, the neglected features are physically important. Relying on a simplified model, it is not clear how strongly the approximations influence the magnetic thermodynamics. (c) The treatment of the $\mathrm{Rh}$ moment as induced gives two terms to the model Hamiltonian: the bilinear term, which describes the energy of the interaction of the $\mathrm{Fe}$ and $\mathrm{Rh}$ moments, and the on-site term, which gives an additional energy contribution because of the variation of the value of the Rh moment. In the case of FM, this treatment changes the number of the magnetic degrees of freedom. The two terms generate opposite trends and compensate each other considerably. In the case of AFM, the account for the induced $\mathrm{Rh}$ moment decreases the Neel temperature, whereas in the case of the FM the Curie temperature increases. (d) It is important to develop a model that treats both the $\mathrm{Fe}$ and $\mathrm{Rh}$ moments as degrees of freedom and that deals in a consistent way with the fluctuations of the values and directions of the $\mathrm{Rh}$ moments. Up to now we have not considered the longitudinal fluctuations of the atomic moments, although the general approach of constrained calculations formulated in Sec. III B allows for this: The longitudinal fluctuations correspond to a constraint with effective fields collinear to the directions of the moments. Such calculations have been recently performed for $\mathrm{NiMnSb}^{37}$ and have shown their importance. In the next section we consider one type of such states to, first, emphasize their relevance for the thermodynamics of FeRh and, second, to discuss their importance for the femtomagnetic phenomena. (e) To describe the AFM-FM phase transition, the energy functions must not only properly describe the energetics of 

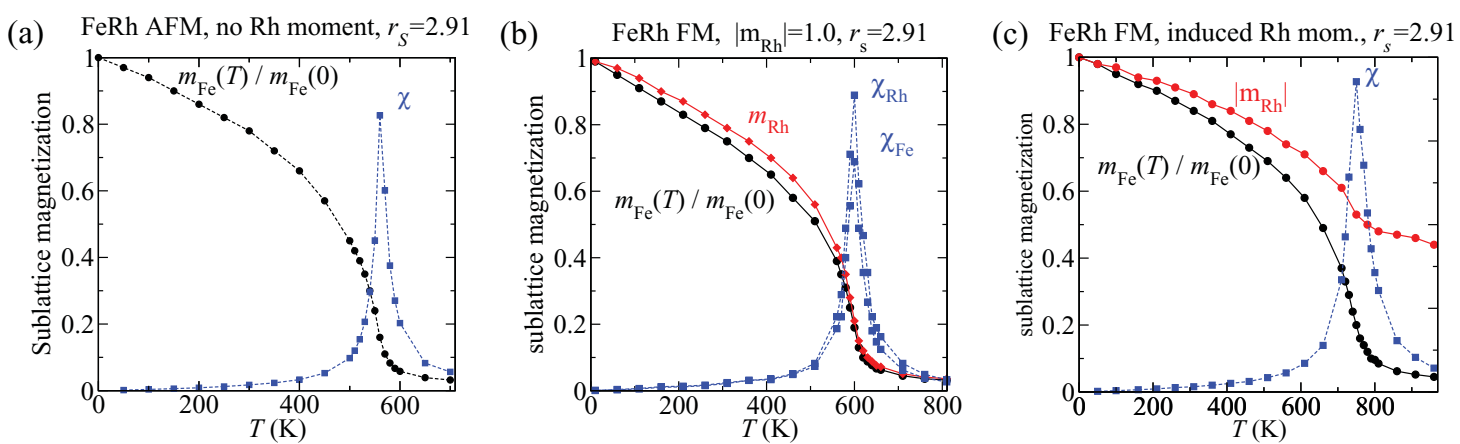

FIG. 11. (Color online) The results of the Monte Carlo simulations performed with the Fe-Fe exchange parameters for $r_{S}=2.91$ a.u. The magnetization curves corresponding to Fe give the magnetization in units of the Fe atomic moment, whereas the data related to the Rh magnetic moment are in units of $\mu_{B}$. Magnetic susceptibilities are given in arbitrary units. (a) The generation of the Rh moment is not taken into account. The system is AFM. The magnetization curve gives the sublattice magnetization. (b) The results of the Monte Carlo simulation for the FM phase of FeRh. Both Fe and Rh moments are treated as Heisenberg degrees of freedom. The value of the Rh moment has a constant value of $1.0 \mu_{B}$. (c) Same as in (b) but with the Rh moments treated as induced by the Fe moments and not constituting additional degrees of freedom. The magnetization curve of the Rh moments coincides, in this case, with the plotted Fe curve. The curve labeled with $\left|m_{\mathrm{Rh}}\right|$ gives the average of the absolute value of the Rh moments.

the regions of the phase space corresponding to each of these phases but also the relative energies of the AFM and FM states and the energetics of the intermediate part of the phase space serving as the barrier between AFM and FM regions. Our calculations show that the LSDA does not provide the correct energy landscape for the equilibrium volume. $\mathrm{Gu}$ and Antropov suggest including nonlocal corrections to improve the energy functions. A systematic study of the performance of various functionals is highly desirable and would provide a useful tool for the development of improved DFT schemes for the description of magnetic systems.

\section{LONGITUDINAL CONSTRAINT ON Rh MOMENTS}

In the previous section we have seen that the way the excited magnetic states are selected and treated strongly influences the calculated magnetic thermodynamics. We also emphasized that from the general principles of the DFT approach to magnetic excitations there is no basis for suppressing the parameters of the $\mathrm{Rh}$ moments as degrees of freedom. In this section we further develop this idea by demonstrating the importance of the excited states determined by the constraint on the values of the $\mathrm{Rh}$ moments. Such states appear neither in the model by $\mathrm{Ju}$ et $a$. $^{13,22}$ nor in the model by $\mathrm{Gu}$ and Antropov. ${ }^{21}$

Similarly to the calculations shown in Fig. 7, we start with the ground-state AFM configuration assuming the Fe moments to be collinear to the $x$ axis. The condition is then applied where the $\mathrm{Rh}$ moment must assume a given value, $\left(0,0, m_{z}\right)$. The corresponding magnetic state is stabilized by a constraining effective magnetic field, $\left(0,0, h_{z}\right)$, acting on the $\mathrm{Rh}$ atom, that is determined in a self-consistent DFT procedure. ${ }^{38}$ These calculations show that the appearance of the Rh moment leads immediately to a canting of the Fe moments. The results of the calculations for two different volumes are presented in Fig. 12. For convenience of comparison with Fig. 7, the abscissa gives the value of angle $\theta$, although this is not an independent variable as in the calculations discussed in Sec. III B but a calculated quantity.
The similarity between data obtained with different constraints is remarkable (Figs. 7 and 12). The values of the Fe moments show very weak $\theta$ dependence. The value of the $\mathrm{Rh}$ moment is roughly proportional to the cosine of the angle between $\mathrm{Rh}$ and $\mathrm{Fe}$ moments. The scale of the energy variation again depends dramatically on the atomic volume.

Thus, in the same way as the canting of the Fe moments from the AFM state leads to the appearance of "induced" $\mathrm{Rh}$ moment, the longitudinal fluctuation of the $\mathrm{Rh}$ moment results in the canting of the Fe moments. The physical reason for both processes is a strong $\mathrm{Fe}-\mathrm{Rh}$ wave-function hybridization

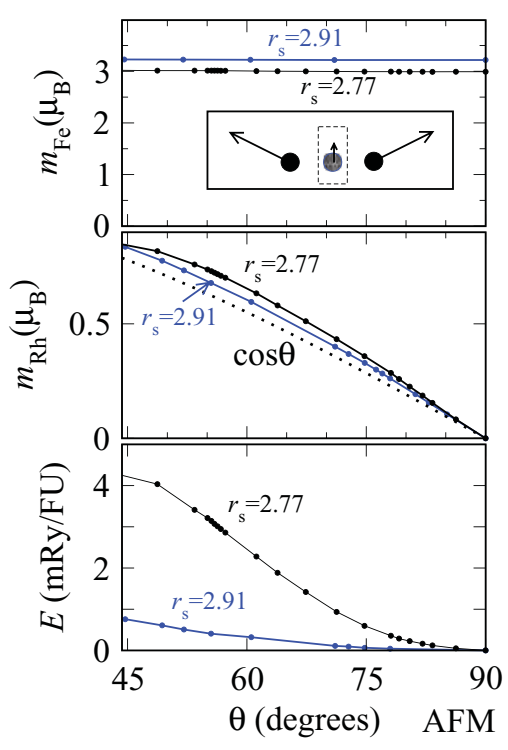

FIG. 12. (Color online) The results of constrained calculations with constraint imposed on the value of the Rh moment for $r_{S}=$ 2.77 a.u. and $r_{S}=2.91$ a.u.. The upper panel gives the value of the Fe magnetic moment and the schematic picture of the magnetic configurations used in the calculations. The middle panel presents the values of the $\mathrm{Rh}$ moments and, for comparison, the cosine function. The bottom panel shows the energies of the magnetic configurations. 
making the correlated behavior of the $\mathrm{Rh}$ and Fe moments energetically advantageous. It is worth stressing, however, that although the underlying physical mechanism shaping the two types of excitations is the same, these are two different excited states characterized by different constraints and, therefore, different spin densities.

In a consequent statistical mechanics treatment, both types of states must be taken into account. Also, in the femtomagnetic experiments, both types of states are expected to contribute to the appearance of the net magnetization (see Sec. VI).

It is useful to remark that in the statistical mechanics of the equilibrium state the contribution of an excited state is determined by the energy of the state. The situation is more complex in the femtomagnetic effects where the system is not in equilibrium and the speed of angular momentum transfer between subsystems involved is a decisive characteristic. Within the Elliott-Yafet mechanism the efficiency of the angular-momentum transfer is determined by the spin-orbit coupling effects that are discussed in Sec. VI.

\section{EFFECTS OF SPIN-ORBIT COUPLING}

Spin-orbit coupling lowers the symmetry of the quantummechanics problem and results in a number of new effects. First, the spin projection of the electron states ceases to be a good quantum number. The states now contain contributions with both spin projections. This feature is essential for the Elliott-Yafet mechanism of the spin-flip electron scattering by the lattice vibrations. ${ }^{10,11}$ In magnetic crystals where the time-reversal invariance is no longer present, the spin-orbit coupling breaks the symmetry between atomic orbitals with opposite magnetic quantum numbers, $+m$ and $-m$, and leads to the formation of nonzero atomic orbital moments. Through interatomic hybridization this property can be transferred from one atom to another and lead to nonzero orbital moment even at atoms with zero spin-orbit coupling. Therefore it is instructive to perform a computational experiment by switching off the spin-orbit coupling on one of the atomic species.

We begin by examining the calculated orbital moments (Table I). At an atomic volume corresponding to $r_{S}$ $=2.77$ a.u., the orbital moment of Fe is found to be $\sim 0.08 \mu_{B}$ in both the AFM and FM states. This value is of the order of the

TABLE I. Orbital moments in FeRh (in $\mu_{B}$ ). First column: magnetic structure of the system. Second column: atom types for which the spin-orbit coupling (SOC) was taken into account. Third and fourth columns: orbital moments of Fe and Rh atoms, respectively. The crystal volume in the calculations corresponds to $r_{S}=2.77$ a.u..

\begin{tabular}{lccc}
\hline \hline & SOC & $m_{\text {orb }}(\mathrm{Fe})$ & $m_{\text {orb }}(\mathrm{Rh})$ \\
\hline FM & Fe,Rh & 0.078 & 0.066 \\
FM & Fe & 0.063 & 0.005 \\
FM & Rh & 0.017 & 0.060 \\
AFM & Fe,Rh & 0.080 & 0 \\
AFM & Fe & 0.066 & 0 \\
AFM & Rh & 0.015 & 0 \\
\hline \hline
\end{tabular}

atomic orbital moment of bulk bcc-Fe. ${ }^{39,40}$ The orbital moment of the $\mathrm{Rh}$ atom is nonzero only for the FM structure and has a value of $\sim 0.07 \mu_{B}$. With the spin-orbit coupling switched off on the Rh atoms we obtained a sizable decrease of the $\mathrm{Fe}$ orbital moment and very small value of the Rh orbital moment (Table I). In the calculations with the spin-orbit coupling switched off on the Fe atom, the Rh orbital moment decreases weakly, whereas the Fe moment still preserves a sizable value of $0.02 \mu_{B}$ (Table I). It is also worth noting that although the spin moment of the $\mathrm{Rh}$ atoms is three times smaller than the spin moment of the Fe atoms the values of the orbital moments of the two atomic species are close to each other. Altogether, these calculations show the importance of the spin-orbit coupling of the $\mathrm{Rh}$ atom.

Next, we estimate the strength of the spin mixture caused by the spin-orbit coupling. This characteristic varies strongly from state to state and depends sensitively on the detailed properties of the electronic structure. For instance, an energetic proximity of states with opposite spin projections leads to a strong spin-mixing under influence of the spin-orbit coupling. For a given electron state the strength of the spin mixture is characterized by the smaller of the two spin components, ${ }^{6}$ yielding a spin-mixing parameter

$$
\left\langle b^{2}\right\rangle=\overline{\min \left(\left\langle\psi_{\mathbf{k}} \mid \uparrow\right\rangle\left\langle\uparrow \mid \psi_{\mathbf{k}}\right\rangle,\left\langle\psi_{\mathbf{k}} \mid \downarrow\right\rangle\left\langle\downarrow \mid \psi_{\mathbf{k}}\right\rangle\right)} .
$$

The line over the right-hand side of the formula denotes averaging over the states. In the femtomagnetic relaxation processes following the laser excitation of the system the states in a certain energy interval about the Fermi energy are involved. We performed the calculation of parameter $\left\langle b^{2}\right\rangle$ for the FM phase and for a number of energy intervals about the Fermi energy. The results of the calculations are collected in Table II. We obtained a relatively weak dependence of the parameter on the size of the energy interval.

As was done above for the orbital moment, we can again verify the importance of the spin-orbit coupling of each of the atom types by successive switching off the spin-orbit coupling for different atoms (Table II). Remarkably, switching off the spin-orbit coupling for Fe even increases somewhat calculated $\left\langle b^{2}\right\rangle$, whereas switching off the spin-orbit coupling for $\mathrm{Rh}$ leads to sizable decrease of the parameter. We conclude that the spin-orbit coupling on both atoms contributes importantly to the spin mixing although the spin-orbit coupling of $\mathrm{Rh}$ appears as more efficient mixing interaction. This is expected,

TABLE II. Elliott-Yafet spin-mixing parameter for FM FeRh integrated over different energy intervals $\left[E_{F}-\Delta E, E_{F}+\Delta E\right]$ about the Fermi energy. Three cases are considered: the spin-orbit coupling for both $\mathrm{Fe}$ and $\mathrm{Rh}$ atoms and the spin-orbit coupling on one of the atomic types. Presented are the results for atomic radius $r_{S}=2.77$ a.u..

\begin{tabular}{lccc}
\hline \hline & \multicolumn{3}{c}{$\left\langle b^{2}\right\rangle$} \\
\cline { 2 - 4 }$\Delta E(\mathrm{mRy})$ & SOC Fe,Rh & SOC Fe & SOC Rh \\
\hline 5 & 0.027 & 0.018 & 0.029 \\
10 & 0.025 & 0.014 & 0.027 \\
50 & 0.020 & 0.011 & 0.023 \\
100 & 0.025 & 0.010 & 0.027 \\
\hline \hline
\end{tabular}


since the spin-orbit coupling strength rises, as a rule of thumb, with the square of the atomic number, so Rh should be a much more efficient spin-orbit scatterer than Fe.

Our value of the $\left\langle b^{2}\right\rangle$ parameter is of the same order as the estimation for the $3 d$ ferromagnets calculated by Steiauf and Fähnle, ${ }^{6}$ which was found to be sufficiently large to explain the ultrafast demagnetization in the $3 d$ metals. Another factor of the Koopmans et al. ${ }^{5}$ theory to determine the efficiency of the Elliott-Yafet mechanism is the ratio of the Curie temperature to the value of atomic moment. In FeRh, $T_{C} \approx 670 \mathrm{~K}$. To perform a rough estimation, we introduce effective values of the atomic moments in FeRh $\sqrt{m_{\mathrm{Fe}} \cdot m_{\mathrm{Rh}}} \approx 1.7 \mu_{B}$, so the energy of the Fe-Rh remains unchanged: $J_{\mathrm{FeRh}} \mathbf{m}_{\mathrm{Fe}}^{i} \cdot \mathbf{m}_{\mathrm{Rh}}^{v}=J_{\mathrm{FeRh}} \mathbf{m}_{\mathrm{eff}}^{i} \cdot \mathbf{m}_{\mathrm{eff}}^{v}$ The ratio $T_{C} / m_{\text {eff }} \sim 376$ is about 3 times smaller than in $\mathrm{Ni}, 627 / 0.62 \sim 1011$ but more than 9 times larger than the corresponding estimation for Gd, 297/7.55 39. Since the theory by Koopmans et al. contains an unknown multiplier expected to vary between 1 and 10, no quantitative comparison of the efficiency of the Elliott-Yafet mechanism in different systems is possible. On a qualitative level, the estimations of the spin-mixing parameter $\left\langle b^{2}\right\rangle$ and the ratio $T_{C} / m_{\text {eff }}$ support the conclusion that the Elliott-Yafet mechanism plays an important role in the femtomagnetism of FeRh.

The calculation of the spin mixing caused by the spin-orbit coupling in the case of AFM is less straightforward because of the degeneracy of the states with opposite values of the spin projection on the quantization axis. To lift this degeneracy we performed the calculations in small external field. The resulting spin-mixing parameter $b^{2}$ was found to be similar to the values obtained for the FM case.

Our analysis suggests two possible processes leading to the ultrafast generation of the magnetization of FeRh. First, by means of the Elliott-Yafet mechanism the electrons excited by the laser light experience scattering on the lattice vibrations resulting in the formation of the spin moment of the $\mathrm{Rh}$ atoms. This, by means of strong Rh-Fe hybridization, leads to the canting of the Fe moments resulting in a magnetized state of FeRh. Second, the electron scattering leads to the canting of the Fe moments and by means of the Rh-Fe hybridization to a nonzero Rh moment.

On the qualitative level of our discussion it is not possible to judge on the relative importance of the two processes leading to the generation of net magnetization. A quantitative study of the spin relaxation by means of the Elliott-Yafet mechanism needs detailed account for the shape of the electron wave functions as well as of the eigenstates of the lattice vibrations. The scattering probability of the electron states on phonons is determined by matrix elements comprising all this infrormation. Because of the presence of two different atoms in $\mathrm{FeRh}$, the $\mathrm{Fe}$ and $\mathrm{Rh}$ sublattices will, in general, provide different contributions to electron and phonon eigenstates. Also spin-mixing for a given electron state, caused by the spin-orbit coupling, differs for $\mathrm{Fe}$ and $\mathrm{Rh}$ contribution to the state. The quantitative study of the Elliott-Yafet scatterring processes in $\mathrm{FeRh}$ is an important task for the future research.

An interesting, in this respect, experimental result was recently reported by Boeglin et al.. ${ }^{9}$ Using time-resolved X-ray magnetic circular dichroism (XMCD) measurements on ferromagnetic CoPd films, they came to the conclusion that there is no efficient momentum transfer between Co and $\mathrm{Pd}$ in the ultrashort time scale. On the other hand, a static experimental XMCD study on FeRh led to the conclusion about a strong connection between the values of the Fe and $\mathrm{Rh}$ net moments in a broad temperature interval. ${ }^{41}$ A time-resolved XMCD study of the ultrafast dynamics of the $\mathrm{Fe}$ and $\mathrm{Rh}$ magnetization is of strong interest.

\section{CONCLUSIONS}

We have shown that the formation of the $\mathrm{Rh}$ moment under AFM-FM transformation cannot be described within the Stoner picture. Instead, the Rh states are implicitly spinpolarized already in the AFM state. The implicit spin splitting is a consequence of the strong hybridization between the $\mathrm{Fe}$ and $\mathrm{Rh}$ states that is a very important factor in the physics of FeRh. Due to this hybridization, a canting of the Fe moments leads to a redistribution of the locally spin-up and spin-down components of the $\mathrm{Rh}$ states and to a net $\mathrm{Rh}$ moment.

We have demonstrated that the ferromagnetic Fe-Rh exchange interaction is robust with respect to the crystal volume variation, whereas the antiferromagnetic $\mathrm{Fe}-\mathrm{Fe}$ exchange interaction is strongly volume dependent. These different volume dependencies of the competing exchange interactions lead to their strong compensation at certain crystal volume. This compensation makes the AFM and FM states in FeRh close in energy and influences strongly the physics of the system. We have also shown that the choice of the magnetic degrees of freedom influences the calculated thermodynamics.

We have further pointed out that the excited states resulting from the variation of the value of the $\mathrm{Rh}$ moment considered as degree of freedom are important for both equilibrium thermodynamics of FeRh and femtomagnetic phenomena in this system. We have estimated the Elliott-Yafet spinmixing parameter and obtained values comparable with earlier calculations for the ferromagnetic $3 d$ metals. We have drawn the conclusion that the Elliott-Yafet mechanism of the angularmomentum transfer between electrons and lattice plays an important role in the femtomagnetic properties of FeRh.

\section{ACKNOWLEDGMENTS}

L.S. is greatful to Stefan Blügel for hospitality during his visits to Jülich. P.M. wishes to thank Stefan Blügel for his support during the project.

\footnotetext{
*1sandr@mpi-halle.de

${ }^{1}$ Uwe Bovensiepen, Nat. Phys. 5, 461 (2009).

${ }^{2}$ J. Stöhr and H.-C. Siegmann, Magnetism: From Fundamentals to

Nanoscale Dynamics (Springer, Berlin, 2006)
}

${ }^{3}$ M. S. Si and G. P. Zhang, J. Phys. Condens. Matter 22, 076005 (2010).

${ }^{4}$ C. Stamm, N. Pontius, T. Kachel, M. Wietstruk, and H. A. Dürr, Phys. Rev. B 81, 104425 (2010). 
${ }^{5}$ B. Koopmans, G. Malinowski, F. Dalla Longa, D. Steiauf, M. Fähnle, T. Roth, M. Cinchetti, and M. Aeschlimann, Nat. Mater. 9, 259 (2010).

${ }^{6}$ D. Steiauf and M. Fähnle, Phys. Rev. B 79, 140401 (2009).

${ }^{7}$ M. Cinchetti, M. Sánchez Albaneda, D. Hoffmann, T. Roth, J.-P. Wüstenberg, M. Krauß, O. Andreyev, H. C. Schneider, M. Bauer, and M. Aeschlimann, Phys. Rev. Lett. 97, 177201 (2006).

${ }^{8}$ G. P. Zhang and W. Hübner, Phys. Rev. Lett. 85, 3025 (2000).

${ }^{9}$ C. Boeglin, E. Beaurepaire, V. Halte, V. Lpez-Flores, C. Stamm, N. Pontius, H. A. Dürr, and J.-Y. Bigot, Nature 465, 458 (2010).

${ }^{10}$ R. J. Elliott, Phys. Rev. 96, 266 (1954).

${ }^{11}$ Y. Yafet, in Solid State Physics, edited by F. Seitz and D. Turnbull (Academic, New York, 1963), Vol. 14.

${ }^{12}$ M. Battiato, K. Carva, and P. M. Oppeneer, Phys. Rev. Lett. 105, 027203 (2010).

${ }^{13}$ Ganping Ju, Julius Hohlfeld, Bastiaan Bergman, Rene J. M. van deVeerdonk, Oleg N. Mryasov, Jai-Young Kim, Xiaowei Wu, Dieter Weller, and Bert Koopmans, Phys. Rev. Lett. 93, 197403 (2004).

${ }^{14}$ Jan-Ulrich Thiele, Matthias Buess, and Christian H. Back, Appl. Phys. Lett. 85, 2857 (2004).

${ }^{15}$ G. Shirane, R. Mathans, and C. W. Chen, Phys. Rev. 134, A1547 (1964).

${ }^{16}$ B. Koopmans, M. van Kampen, and W. J. M. de Jonge, J. Phys. Condens. Matter 15, S723 (2003).

${ }^{17}$ M. Fallot, Ann. Phys. (Paris) 10, 291 (1938).

${ }^{18}$ V. L. Moruzzi and P. M. Marcus, Phys. Rev. B 46, 2864 (1992).

${ }^{19} \mathrm{~J}$. Kübler, Theory of Itinerant Electron Magnetism (Oxford University Press, Oxford, 2000).

${ }^{20}$ M. E. Gruner, E. Hoffmann, and P. Entel, Phys. Rev. B 67, 064415 (2003).

${ }^{21}$ R. Y. Gu and V. P. Antropov, Phys. Rev. 72, 012403 (2005).

${ }^{22}$ O. N. Mryasov, Phase Trans. 78, 197 (2005).

${ }^{23}$ C. Kittel, Phys. Rev. 120, 335 (1960).

${ }^{24}$ J. B. McKinnon, D. Melville, and E. W. Lee, J. Phys. C 3, S46 (1970).

${ }^{25}$ A. R. Williams, J. Kübler, and C. D. Gelatt, Phys. Rev. B 19, 6094 (1979).
${ }^{26}$ The SPR-TB-KKR package, H. Ebert and R. Zeller [http://olymp.cup.uni-muenchen.de/ak/ebert/SPR-TB-KKR].

${ }^{27}$ S. H. Vosko, L. Wilk, and M. Nusair, Can. J. Phys. 58, 1200 (1980).

${ }^{28}$ P. H. Dederichs, S. Blügel, R. Zeller, and H. Akai, Phys. Rev. Lett. 53, 2512 (1984).

${ }^{29}$ A. Liechtenstein, M. I. Katsnelson, V. P. Antropov, and V. A. Gubanov, J. Magn. Magn. Mater. 67, 65 (1987).

${ }^{30}$ L. M. Sandratskii, Phys. Rev. B 64, 134402 (2001).

${ }^{31}$ The value of the Stoner parameter is obtained as the ratio of the exchange splitting of the atomic $4 d$ level to the value of the $\mathrm{Rh}$ moment. The atomic levels are determined by solving the radial Schrödinger equation in a single atomic sphere. The solution of the radial equation in atomic sphere is a part of the calculation machinery for a solid.

${ }^{32}$ David C. Langreth and M. J. Mehl, Phys. Rev. Lett. 47, 446 (1981).

${ }^{33}$ L. M. Sandratskii, R. Singer, and E. Sasioglu, Phys. Rev. B 76, 184406 (2007).

${ }^{34}$ N. Metropolis, A. Rosenbluth, M. Rosenbluth, A. Teller, and E. Teller, J. Chem. Phys. 21, 1087 (1953).

${ }^{35}$ For completeness we should mention that the ground state in this case is a canted noncollinear structure that is an artifact of the nonzero $\mathrm{Rh}$ moment at $0 \mathrm{~K}$. The presence of the moment results in the competition between antiferromagnetic $\mathrm{Fe}-\mathrm{Fe}$ and ferromagnetic Fe-Rh-Fe exchange intercations.

${ }^{36}$ Kouvel and C. C. Hartelius, J. Appl. Phys. 33, 1343 (1962).

${ }^{37}$ L. M. Sandratskii, Phys. Rev. B 78, 094425 (2008).

${ }^{38}$ For convergence purposes it can be more convenient to fix a constraining field $\left(0,0, h_{z}\right)$ and calculate in the self-consistent DFT procedure the value of the $\mathrm{Rh}$ moment $\left(0,0, m_{z}\right)$ and angle $\theta$ between $\mathrm{Fe}$ and $\mathrm{Rh}$ moments.

${ }^{39}$ S. Chadov, J. Minar, M. I. Katsnelson, H. Ebert, D. Ködderitzsch, and A. I. Lichtenstein, Europhys. Lett. 82, 37001 (2008).

${ }^{40}$ W. D. Brewer, A. Scherz, C. Sorg, H. Wende, K. Baberschke, P. Bencok, and S. Frota-Pessoa, Phys. Rev. Lett. 93, 077205 (2004).

${ }^{41}$ C. Stamm, J.-U. Thiele, T. Kachel, I. Radu, P. Ramm, M. Kosuth, J. Minar, H. Ebert, H. A. Dürr, W. Eberhardt, and C. H. Back, Phys. Rev. B 77, 184401 (2008). 